\title{
Modelling and forecasting of quasi-periodic processes in technical objects based on cylindrical image models
}

\author{
V R Krasheninnikov ${ }^{1}$, Yu E Kuvayskova ${ }^{1}$ \\ ${ }^{1}$ Ulyanovsk State Technical University, Severny Venets street, 32, Ulyanovsk, Russia, 432027
}

e-mail: kvrulstu@mail.ru, u.kuvaiskova@mail.ru

\begin{abstract}
Accurate forecasting of the state of technical objects is necessary for effective management. The technical condition of the object is characterized by a system of time series of monitored indicators. The time series often have difficultly predictable irregular periodicity (quasi-periodicity). In this paper, to improve the accuracy of such series forecasting, models of quasi-periodic processes in the form of samples of a cylindrical image are used. The application of these models is demonstrated by forecasting of a hydraulic unit vibrations. It is shown that the use of these models provides a higher accuracy of prediction compared with the classical approaches.
\end{abstract}

\section{Introduction}

In order to improve the management of a technical object an accurate forecast of its technical condition is required, which is characterized by a variety of controlled indicators. These indicators are recorded by sensors at certain time intervals in the form of signals with discrete time. These signals can be used to predict state of an object in order to make effective management decisions.

The set of registered values of the object's indicators can be represented as a system of time series. To represent the time series, a large number of models have been developed: autoregressive, spectral, wave, wavelets, and so on, for example, [1-4]. A very important task is the prediction of time series, which is associated with the management of objects. To solve this problem, the methods of mathematical statistics, artificial neural networks, fuzzy logic, machine learning and so on are used, for example, in [5-14].

Often time series have irregular periodicity, that is, quasi-periodicity. Quasi-periodic behavior is a repetition with a component of unpredictability. For example, the vibration of various engines, turbines, units and so on.

Different models can be used to describe and predict such processes, for example, autoregressive models with complex roots of the characteristic equation or harmonic models. However, these models do not always allow to predict a quasi-periodic process with high accuracy. In this paper, to improve the accuracy of predicting the state of technical objects, it is proposed to use models of quasi-periodic processes based on cylindrical images [15-18].

\section{Quasi-periodic process as a scan of an image}

Double correlation is a characteristic property of a quasi-periodic process, that is, there is a strong correlation both between adjacent values and between values separated by several periods.

There are a number of approaches to describe quasi-periodicity: the imposition of noise or higher frequencies on the main harmonic, periodic nonstationarity (fluctuation of moments and other characteristics), and so on. A common manifestation of such representations is the high correlation of process values at distances that are multiples of a period. In this paper,this property is 
taken as a basis, that is, as the main property of a quasiperiodic process.

As quasi-periods of the process, we could take the lines of a certain rectangular image: the required correlation will be ensured by the vertical correlation of the image. Consider, for example, an image on a rectangle, given by the Habibi model [19]:

$$
x_{k, l}=a x_{k, l-1}+b x_{k-1, l}-a b x_{k-1, l-1}+\beta \sqrt{\left(1-a^{2}\right)\left(1-b^{2}\right)} \xi_{k, l},
$$

where $\mathrm{k}$ is the line number, 1 is the column number, $\xi_{k, l}$ is a set of independent standard random variables. The generated image has a covariance function $(\mathrm{CF})$

$$
V(m, n)=M\left[x_{k, l} x_{k+m, l+n}\right]=\beta^{2} a^{|m|} b^{|n|} .
$$

Parameters $a$ and $b$ affect the vertical and horizontal image correlation, respectively; $\beta$ affects the variance of the image. Figure 1 shows an example of simulated image.

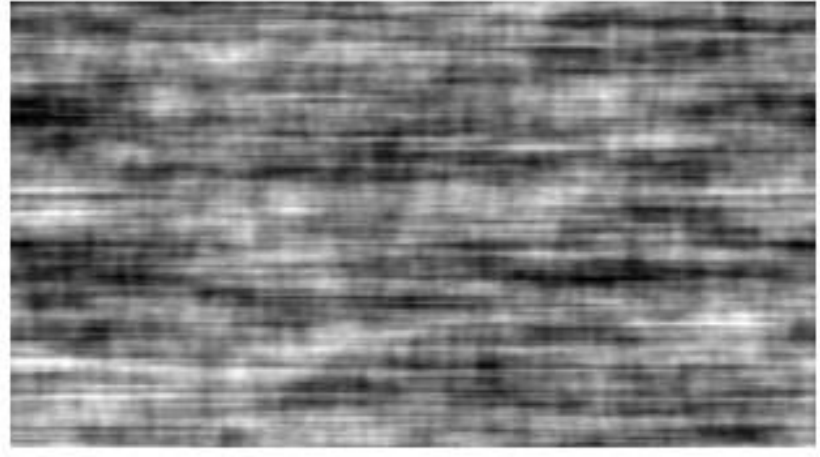

(a)

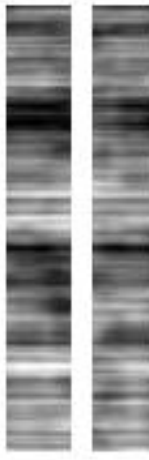

(b)

Figure 1. Image simulated by model (1): (a) full image, (b) the first and last five columns.

Image rows are highly correlated if $b \approx 1$. Therefore, by combining the rows into a sequence, we can get a model of a quasi-periodic process. Figure 2 shows an example of such process. The boundaries of the periods are indicated by vertical lines, where sharp jumps are noticeable. In fact, this figure shows the brightness graphs of successive rows of the image.

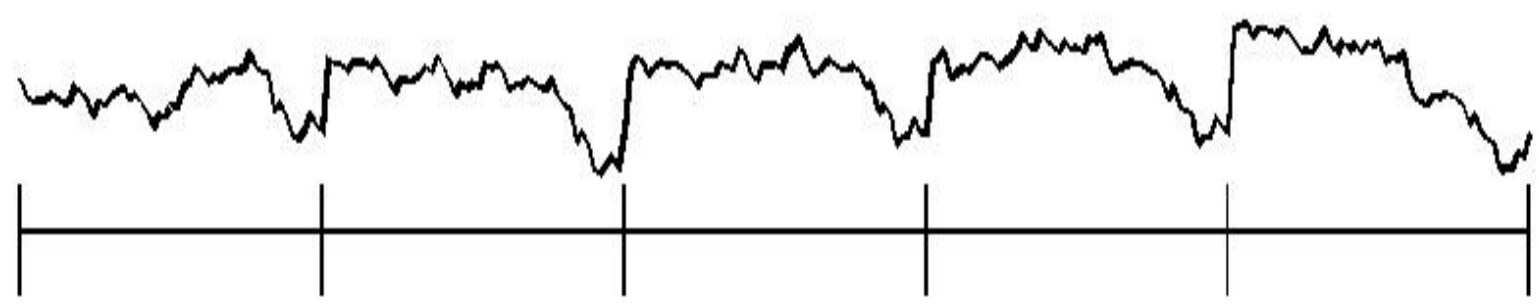

Figure 2. Process simulated using model (1).

The CF of the process is shown in Figure 3. Correlations between elements of the image decrease along the rows. Therefore, the $\mathrm{CF}$ of the process decreases along the period, but increases sharply at the junction of the periods. This property of the $\mathrm{CF}$ just ensures the quasi-periodicity of the process.

However, the beginning and end of each row, being at a considerable distance, are practically independent of each other, which is noticeable in Figure 1, b. Therefore, at the junctions of the quasiperiods of the process obtained by joining the rows, there will be a weak correlation of neighboring values, leading to sharp jumps that are not typical of relatively continuous processes. Similar discontinuity is obtained by combining rows from other rectangular images. Thus, rectangular images 
do not provide acceptable representations of quasi-periodic processes. In this paper, modeling processes using images on a cylinder is considered.

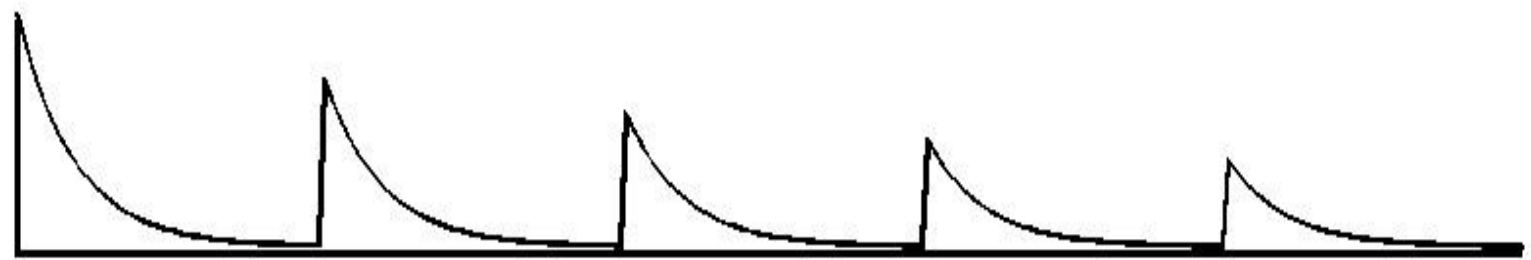

Figure 3. The correlation function of the model (1).

Let's consider a cylindrical image, which is scanned along a spiral on this cylinder (figure 4 (a)). In [15], the following model was used to represent images on a cylindrical grid similar to Habibi autoregression model (1):

$$
x_{k, l}=a x_{k, l-1}+b x_{k-1, l}-a b x_{k-1, l-1}+c \xi_{k, l}
$$

where $k$ is a spiral turn number; $l$ is a node number $l=0, \ldots, T ; x_{k, l}=x_{k+1, l-T}$ when $l \geq T ; T$ is the period, i.e. the number of points in one turn; $\xi_{k, l}$ are independent standard random variables.

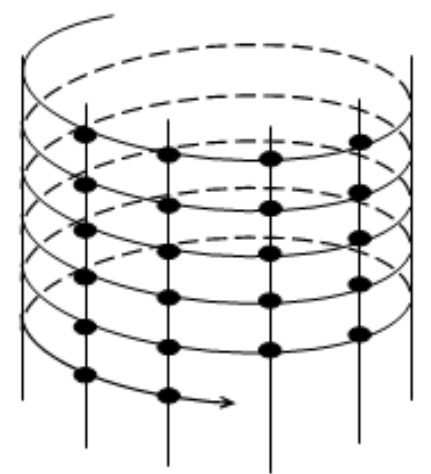

(a)

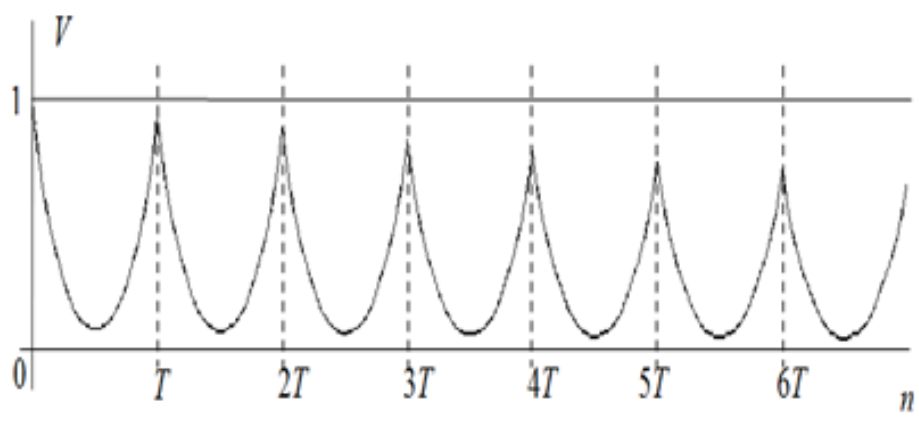

(b)

Figure 4. Cylindrical image: (a) cylindrical spiral, (b) normalized correlation function.

An example of an image simulated using this model is shown in Figure 5 (a). Its first 5 columns are shown in Figure 5 (b). It is noticeable that the first and last columns are strongly correlated.

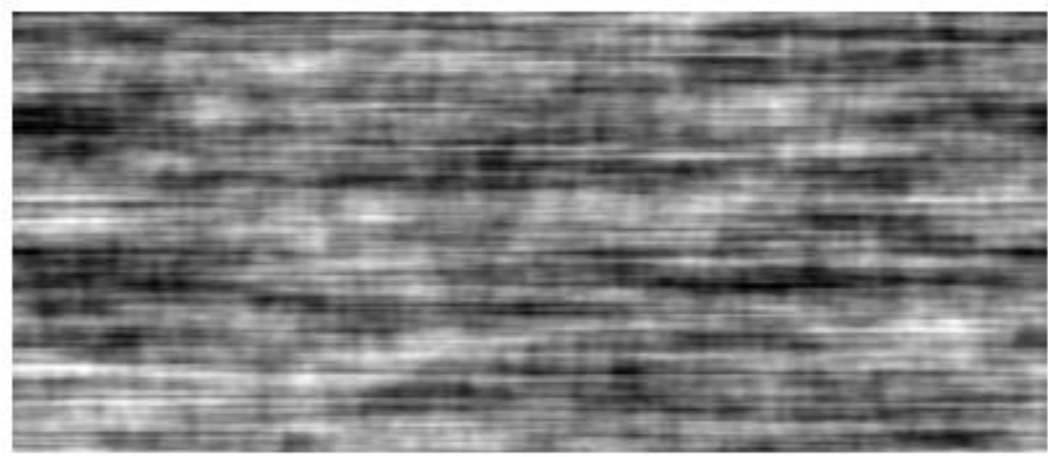

(a)

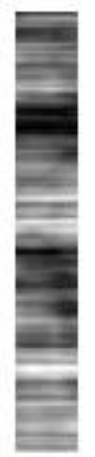

(b)

Figure 5. Simulated cylindrical image (a) and its first columns (b). 
For convenience of analyzing this model, assume that the pixels are numbered and located on a cylindrical spiral (figure 4 (a)). Then model (2) can be represented in an equivalent form as a model of a random process, which is a scan of the image along the spiral:

$$
x_{n}=a x_{n-1}+b x_{n-T}-a b x_{n-T-1}+c \xi_{n},
$$

where $n=k T+l$. Applying the z-transform, we obtain the CF

$$
V(n)=M\left[x_{m} x_{m \pm n}\right]=c^{2}\left(\frac{1}{\left(1-b^{2}\right) T} \sum_{k=0}^{T-1} \frac{z_{k}}{\left(1-a z_{k}\right)\left(z_{k}-a\right)} z_{k}^{n}+\frac{s}{\left(1-a^{2}\right)(1-b s)(s-b)} \rho^{n}\right),
$$

where $z_{k}=\sqrt[T]{b} \exp (i 2 \pi k / T)$ and $s=a^{T}$.

In particular, when $n=m T$ we obtain

$$
V(m T)=\frac{c^{2}}{\left(1-a^{2}\right)\left(1-b^{2}\right)(1-s b)(b-s)}\left(\left(1-s^{2}\right) b^{m+1}-\left(1-b^{2}\right) s^{m+1}\right)
$$

and the variance, when $m=0$ :

$$
\sigma^{2}=\frac{c^{2}(1+b s)}{\left(1-a^{2}\right)\left(1-b^{2}\right)(1-b s)}
$$

To reduce the calculations, it is possible to calculate only $V(0), V(1), \ldots, V(T)$ by formula (4), and for the rest of values, use recurrent formula

$$
V(n)=a V(n-1)+b V(n-T)-a b V(n-T-1) .
$$

This CF (3) decreases with increasing distance $n$, but at distances divisible by period $T, \mathrm{CF}$ is high (figure 4 (b)), which is typical for quasi-periodic processes.

The parameter $a$ affects the correlation along the rows, that is, the smoothness of the process. The parameter $b$ affects the correlation of values over a period distance. For values of $b$ close to 1 , the adjacent rows of the image (spiral turns) will be strongly correlated. In this case the process is closed to periodic one (figure 6(a)). The periodicity property weakens as $b$ decreases (figure 6(b)).

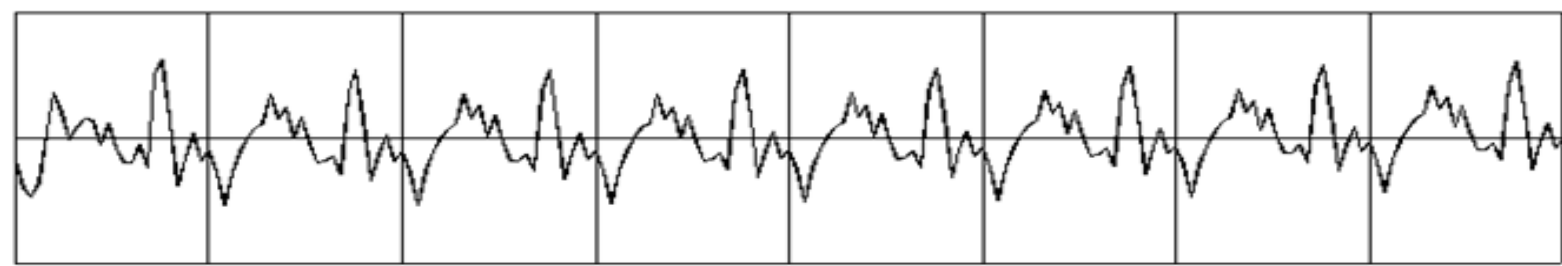

(a)

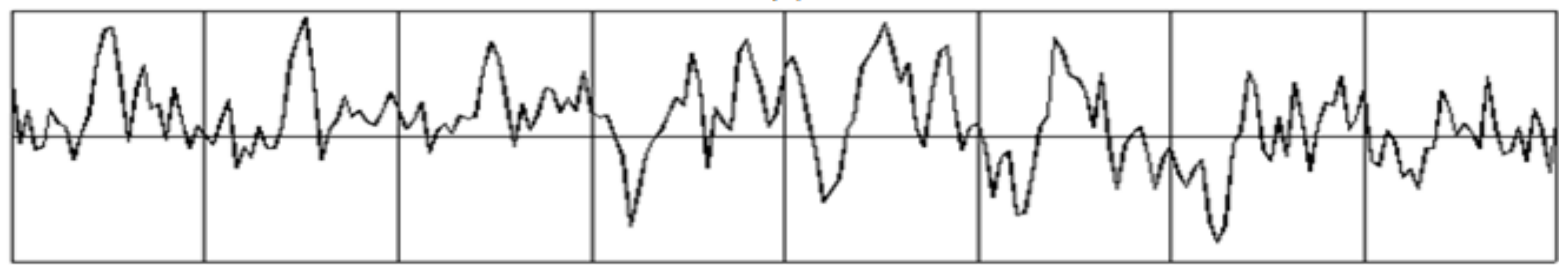

(b)

Figure 6. Simulated processes: (a) $b=0.99$; (b) $b=0.9$. 
We note in particular that these graphs of simulated processes are continuous, in contrast to Figure 2. This is a consequence of the continuity of the CF (4), shown in Figure 4 (b). Thus, model (3) is suitable for describing continuous quasi-periodic processes. By varying the parameters of this model, it is possible to represent processes with a given correlation within quasi-periods and between them.

\section{Prediction of the state of a technical object}

As an object of study, we consider data on a hydraulic unit (one of the turbines of the Krasnaya Polyana Hydroelectric Power Plant, Russia). Figures 7-9 show vibration plots obtained from three sensors located on different parts of the unit. The task was to predict the next process value using its previous values. Visually, the presence of quasi-periodicity in these processes is observed. This makes it possible to apply the model of quasiperiodic processes described above to these data. The volume of each sample was 96 observations.

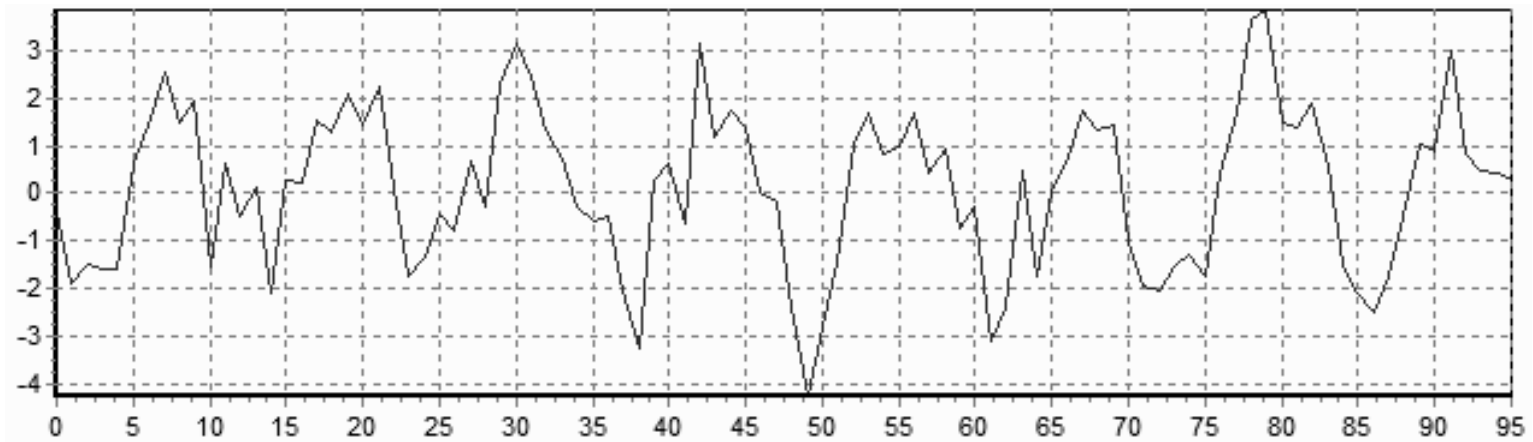

Figure 7. The first process of vibration of the hydraulic unit.

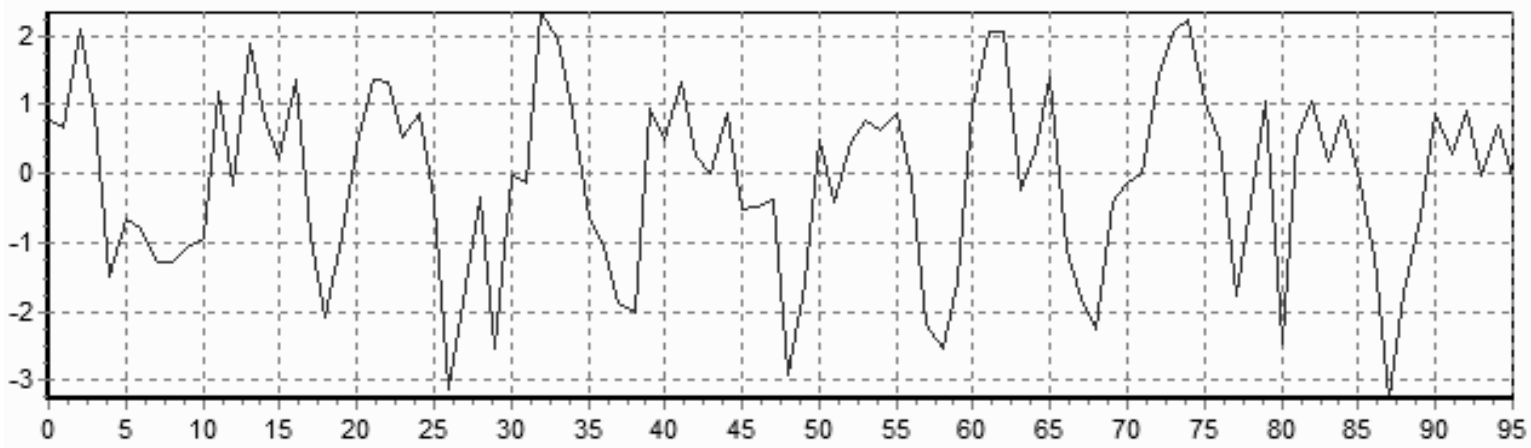

Figure 8. The second process of vibration of the hydraulic unit.

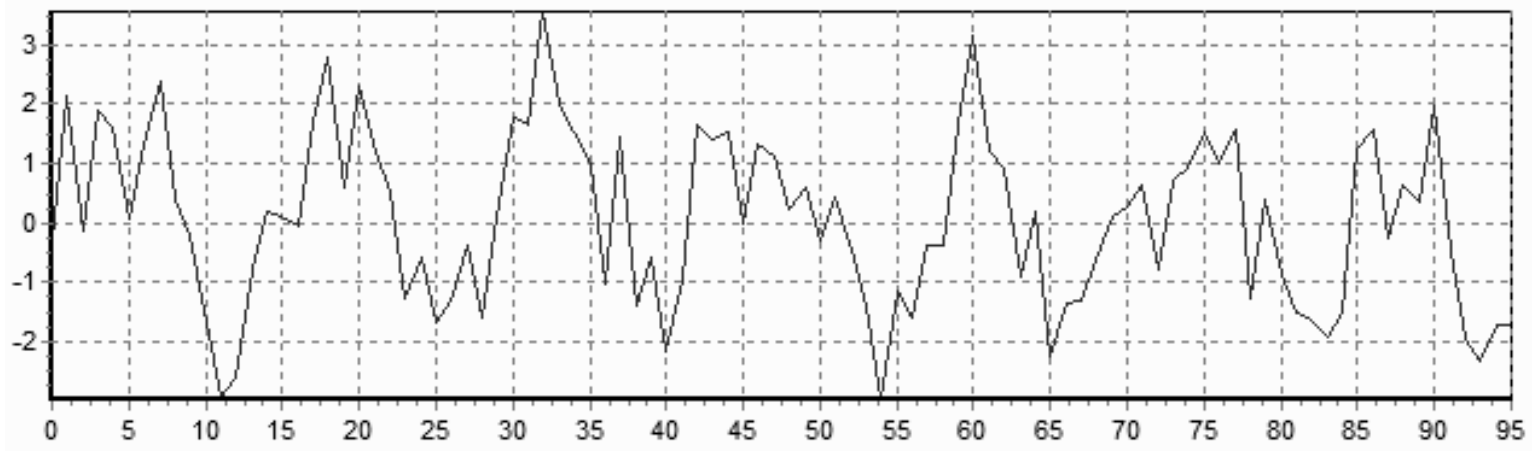

Figure 9. The third process of vibration of the hydraulic unit. 
To assess the quality of forecasting of these processes, the sampling of values is divided into two parts: a training sample (90\% of the volume of initial observations) and a control sample (10\% of the volume of initial observations). According to the training sample, we construct by the least squares method three models of the studied processes: an autoregressive model of a cylindrical image (3), a usual autoregressive model of the second order with complex roots of the characteristic equation and a harmonic model. For the control sample, we estimate the prediction accuracy of the constructed models as $\sigma_{\Delta}=\sqrt{\frac{1}{k} \sum_{n=1}^{k}\left(x_{n}-\hat{x}_{n}\right)^{2}}$, where $k$ is the volume of the control sample; $x_{i}$ are the values of the observation; $\hat{x}_{i}$ are the predicted value of the process according to the constructed model. In the case of a cylindrical model, the equation

$$
\hat{x}_{n}=a x_{n-1}+b x_{n-T}-a b x_{n-T-1}
$$

was used to predict the next process value from its previous values. The results of the comparison of models for prediction accuracy are presented in Table 1.

Table 1. Prediction errors with different models.

\begin{tabular}{lccc}
\hline \multirow{2}{*}{ Process model } & \multicolumn{3}{c}{ Prediction error $\left(\sigma_{\Delta}\right)$} \\
\cline { 2 - 4 } & Process 1 & Process 2 & Process 3 \\
\hline Autoregressive cylindrical image model (2) & 0,6401 & 0,6561 & 0,6676 \\
Second order autoregressive model & 0,7950 & 0,7606 & 0,7756 \\
Harmonic model & 0,9698 & 0,9316 & 0,9532 \\
\hline
\end{tabular}

It follows from this table that the prediction error of the vibrations processes is decreased up to 1.5 times with the use of cylindrical models of images, as compared with the autoregressive and harmonic models.

\section{Conclusions}

It is proposed to use models of quasi-periodic processes in the form of a spiral on a cylindrical image to predict the state of technical objects. The results of numerical experiments show that the use of these models provides a higher prediction accuracy in comparison with the classical time series models.

\section{References}

[1] Anderson T W 1971 The statistical analysis of time series (John Wiley \& Sons Inc. N-Y London Sydney Toronto)

[2] Hannan E J 1970 Multiple time series (John Wiley \& Sons Inc. N-Y London Sydney Toronto)

[3] Jenkins G M and Watts D G 1968 Spectral Analysis and Its Application (San Francisco: GoldenDay) p 525

[4] Daubechies I 1992 Ten lectures on wavelets (Soc Ind Appl Math Philadelphia)

[5] Montgomery D C 2009 Introduction to statistical quality control (New York: John Wiley and Sons) $\mathrm{p} 754$

[6] Montgomery D C, Johnson L A and Gardiner J S 1990 Forecasting and Time Series Analysis (New York: Mc Graw-Hill) p 394

[7] Bahiraee E and Raissi S 2014 Economic design of Hotelling's T2 control chart on the presence of fixed sampling rate and exponentially assignable causes Journal of Industrial Engineering International 10 229-238

[8] Chou C Y and Chen C H 2006 Economic design of variable sampling intervals T2 control charts using genetic algorithms Expert Systems Appl 30 233-242 
[9] Witten I H and Frank E 2005 Data mining: practical machine learning tools and techniques (San Francisco: Morgan Kaufmann Publishers)

[10] Klyachkin V N 2011 Models and methods of statistical control of a multi-parametric process (Moscow: Fizmatlit)

[11] Kuvayskova Y E 2017 The prediction algorithm of the technical state of an object by means of fuzzy logic inference models Procedia Engineering 201 767-772

[12] Yumaganov A S and Myasnikov V V 2017 The method of searching for similar code sequences in executable binary files using the unmarked approach Computer Optics 41(5) 756-784 DOI: 10.18287/2412-6179-2017-41-5-756-764

[13] Kropotov Yu A, Proskuryakov A Yu and Belov A A 2018 A method for predicting changes in the parameters of time series in digital information control systems Computer Optics 42(6) 1083-100 DOI: $10.18287 / 2412-6179-2018-42-6-1083-1092$

[14] Maksimov A I and Gashnikov M V 2018 Adaptive interpolation of multidimensional signals in differential compression Computer Optics 42(4) 679-687 DOI: 10.18287/2412-6179-2018-42-4679-687

[15] Krasheninnikov V R, Kalinov D V and Pankratov Yu G 2001 Autoregressive Model of a Quasiperiodic Signal Pat Rec and Im An 11(1) 211-213

[16] Krasheninnikov V R and Kuvayskova Y E 2016 Prediction of object dynamics using autoregressive models on a cylinder Radio engineering 2016(9) 36-39

[17] Krasheninnikov V R and Subbotin A Yu 2018 Doubly stochastic model of a quasi-periodic process as an image on a cylinder Proceedings of the International Scientific and Technical Conference "Advanced Information Technologies" (Samara: Samara Scientific Center of the Russian Academy of Sciences) 1017-1021

[18] Krasheninnikov V R and Vasil'ev K K 2018 Multidimensional image models and processing Computer Vision in Control Systems-3 ISRL 135 11-64

[19] Habibi A 1972 Two-dimensional Bayesian estimate of images Proc IEEE 60(7) 878-883

[20]Polyak B T and Tsypkin Ya Z 1973 Pseudogradient adaptation and training algorithms Automation and Remote Control 1973(3) 45-68 